\title{
Noise Reduction in Class-AB Microwave-Photonic Links
}

\author{
Thomas E. Darcie ${ }^{1}$, Amol Moye ${ }^{1}$, Peter F. Driessen ${ }^{1}$, \\ Jeffrey D. Bull2, Hiroshi Kato², Nicolas A. F. Jaeger ${ }^{2}$ \\ ${ }^{1}$ University of Victoria, Victoria, British Columbia, Canada, V8W 3P6 \\ ${ }^{2}$ JGKB Photonics, Inc., 221-4664 Lougheed Highway, Burnaby, BC, Canada V5C 5T5
}

\begin{abstract}
A Class-AB microwave-photonic link is presented as a means to reduce noise associated with DCbias. By operating with two complementary, approximately half-wave-rectified, optical sources and generating the difference in a balanced detector, linear modulation with high modulation efficiency is achieved with near zero DC. Using two mode-converter intensity modulators, we demonstrate a $5 \mathrm{~dB}$ reduction in shot noise and elimination of intensity noise, relative to a quadrature-biased MachZehnder, for the same received RF signal power.
\end{abstract}

Index Terms - Microwave-photonic, analog links, subcarrier, low-bias, intensity-noise suppression, class AB.

\section{INTRODUCTION}

Analog or microwave-photonic optical links (MPLs) have found widespread application in numerous sectors of communications, including cable-television and fiberto-the-premises networks, remote antennas, radar links, and phased-array antenna systems. As a result, considerable work has gone into maximizing the performance of these links [1,2]. Noise limits the minimum microwave signal level that can be detected. Linearity limits the maximum signal power that can be transmitted. The difference between minimum and maximum is described by the spur-free dynamic range (SFDR), one of the key figures of merit for an MPL [3].

Normally, the light source (directly-modulated laser or external modulator) is biased to a linear operating point. DC light intensity, or residual carrier, associated with this bias is responsible for many of the limitations of the link performance. Shot noise, relative-intensity noise (RIN), signal-spontaneous beat noise in opticallyamplified systems [4], and impairments from optical nonlinearities (stimulated Brillouin scattering [5], nonlinear refractive index [6], stimulated Raman scattering) are all manifestations of this residual carrier. Also, this residual carrier saturates the photodetector, limiting the maximum signal power. Hence the DC bias required for a linear operating point limits both the low end (noise) and high end (signal power) of the SFDR.

Techniques proposed to mitigate the effect of this residual carrier include optical filtering [7], coherent techniques [8], and dynamic bias modulation [9]. Common-mode RIN can be cancelled using a balanced photodetector [10], but this does not affect the other challenges associated with the large residual carrier. Low-bias techniques have been explored in which a Mach-Zehnder (MZ) is operated at lower bias than the conventional quadrature bias (Q-bias or $\mathrm{QB})$ point $(50 \%$ transmission). Unfortunately, this increases distortion and decreases the signal. To reduce distortion, a novel approach was explored in which $2 \mathrm{MZs}$ were operated in an anti-symmetric manner with a balanced detector [11] Reduction of second-order distortion was demonstrated, but maintaining balance was challenging.

Class-AB Microwave-Photonic Links: With our new approach, we continue along the path suggested in [11], and propose a general class of MPL defined in analogy to Class-AB electronic amplifiers [12]. In Class$\mathrm{AB}$ electronic amplifiers, 2 transistors are operated as a complementary pair, providing independent $(+)$ and (-) signals that are combined to provide a complete signal. Class-AB amplifiers differ from the more ideal Class-B amplifiers by the presence of a small pre-bias that is required to maintain good linearity. In our Class-AB (CAB) MPL, two matched zero- or low-biased sources are operated as a complementary pair, each delivering either $(+)$ or (-) signals. Since optical intensity cannot be less than zero, the zero-mean signal is reconstructed at the output of a balanced detector. DC power in the link, and therefore noise, is minimized. All of the problems described above that are created by the large residual carrier are reduced substantially.

One way to demonstrate the potential merit of the CAB approach is to use a pair of intensity modulators, in the manner described in [11]. However, as their intent was to cancel even-order distortion in a low-biased MZbased link, bias was maintained at a point where evenorder distortion was moderate. It is our intent to maximize SFDR by minimizing noise. Therefore, we operate our modulators at a substantially-reduced bias $(<10 \%$ transmission). This approximately half-wave rectifies the signal, creating large even-order distortion. (In principle, even-order distortion is cancelled, but this requires exact matching.) The microwave signal is reconstructed using a balanced photodetector.

\section{ANALYSIS}

The modulators used here were $40 \mathrm{GHz}$ electro-optic mode converters fabricated in AlGaAs/GaAs. TMpolarized light, coupled into the nominally single-mode channel waveguide comprising the mode converter, is converted to TE polarized light at the modulator's output. This is achieved by electro-optically inducing a phase-shift between the two hybrid modes supported by the waveguide. The output of the mode converter is 
passed through an analyzer aligned to select only TE polarized light. In this way, the mode converter interferes the TE-aligned components of the two supported hybrid modes. In the absence of any intrinsic mode coupling in the device, the output power of the two modulators can be written, as in a conventional MZ, as

$$
\begin{aligned}
& P_{A o}=\frac{L_{e}}{2} P_{i}(1-\cos \pi(v+\Delta v)) \quad \text { and } \\
& P_{B o}=\frac{L_{e}}{2} P_{i}(1-\cos \pi(v-\Delta v)),
\end{aligned}
$$

where $v$ and $\Delta v$ are the modulation and bias voltages normalized to the switching voltage $V_{\pi}, P_{i}$ is the optical power at the input to each modulator, and $L_{e}$ is the excess loss. For a single modulator biased at the conventional quadrature point (QB), $\Delta v=1 / 2$, then

$$
P_{Q o}=\frac{L_{e}}{2} P_{i}(1+\sin \pi v) \text {. }
$$

This results in a detected current in a single detector

$$
I_{Q}=\frac{R_{o} L L_{e}}{2} P_{i}(1+\sin \pi v)=\frac{1}{2} I_{o}(1+\sin \pi v) \text {, }
$$

where $L$ is the loss between the modulator and detector, $R_{o}$ is the detector responsivity $(\mathrm{A} / \mathrm{W})$, and $I_{o}$ is the photocurrent at $100 \%$ modulator transmission.

Considering the system shown in Figure 2 with the bias configuration of Figure 1, for $\mathrm{CAB}$ operation, the current output from the balanced detector can be written: $I_{t}=R_{o} L\left(P_{A o}-P_{B o}\right)=I_{o} \sin \pi v \sin \pi \Delta v$.

Obviously the sum of the two sinusoidal transfer functions is another sinusoid, but with zero average. For small $\Delta v$, the detected signal is small. If $\Delta v=1 / 6$, the detected signal current for $\mathrm{CAB}$ is equal to that of a the single QB link (Equation (4)). This $\pi / 6$ operating point turns out to be a good compromise between large signal power and small residual carrier.

Signal Power: Assuming small-signal modulation of the form $v=v_{o} \cos \omega t$, the root-mean-square (rms) signal power (per ohm) for the $\mathrm{CAB}$ and $\mathrm{QB}$ cases are

$P_{S_{A B}}=\frac{1}{2}\left(I_{o} \pi v_{O} \sin \pi \Delta v\right)^{2}$ and $P_{S_{Q}}=\frac{1}{2}\left(\frac{I_{O} \pi v_{O}}{2}\right)^{2}$.

Shot Noise: Whereas the signal powers from the residual carrier cancel in the balanced detector, the uncorrelated shot noise adds. The total instantaneous (with respect to the RF signal) shot-noise mean-square noise current at the output of the balanced detector is

$$
\sigma_{s}^{2}=2 q R_{o} L\left(P_{A o}+P_{B o}\right) B=2 q I_{o} B(1-\cos \pi v \cos \pi \Delta v) \text {, }
$$

where $q$ is the electronic charge and $\mathrm{B}$, is the noise bandwidth. For small signals and averaging over many RF cycles, the average shot-noise mean-square currents for $\mathrm{CAB}$ and $\mathrm{QB}$ are

$$
{\sigma_{s A B}}^{2}=2 q I_{o} B(1-\cos \pi \Delta v) \text { and } \sigma_{s Q}{ }^{2}=q I_{o} B \text {. }
$$

Equation (8) describes the primary motivation for $\mathrm{CAB}$ operation. Comparing $\mathrm{QB}(\Delta v=1 / 2)$ with $\mathrm{CAB}$ at our exemplary $\Delta v=1 / 6, \mathrm{CAB}$ has a shot-noise reduction of $5.7 \mathrm{~dB}$ for the same RF signal power.

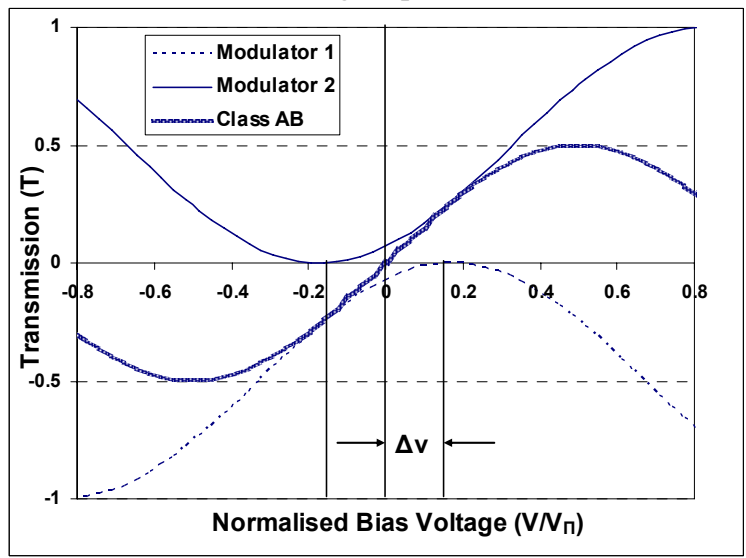

Fig. 1. Normalized transmission of sine-wave reconstruction using complementary biased modulators (Class-AB).

Relative-Intensity (RIN) and Amplifier Noise: Using an optical amplifier prior to the modulator(s) results in signal-spontaneous beat noise that can be described by an additional component to the relativeintensity noise (RIN), described by

$$
R I N^{*}=R I N+2(1-1 / G) F h f / P_{a i},
$$

where $G$ is the amplifier power gain, $F$ the noise figure, $h f$ the photon energy, and $P_{a i}$ is the optical power at the input to the amplifier [13]. It can be shown that the rms noise current from RIN* in a single detector, averaged over multiple RF cycles, is then

$$
\sigma_{R I N^{*}}=I_{o} \sqrt{B \cdot R I N^{*}}(1-\cos \pi \Delta v) / 2 \text {. }
$$

For the single QB modulator, $\cos \pi \Delta v=0$. In determining the total mean-square noise power from RIN*, one can consider the effects of intensity-noise suppression in the balanced detector. This results in

$\sigma_{R I N^{*}}^{2}=I_{o}^{2}\left(B \cdot R I N^{*}\right)(1-\cos \pi \Delta v)^{2} \frac{S^{2}}{4}$.

$S$ is a suppression factor that accounts for the combination of common-mode suppression and impact of modulation [13]. Note that both two-fiber QB and our CAB links can benefit from RIN suppression. However, it can be seen from Equation (11) that $\mathrm{CAB}$ has a large inherent advantage. The ratio of total RIN*-induced noise (for a given $S$ ) for $\mathrm{QB} / \mathrm{CAB}$ is $1 /(1-\cos \pi \Delta v)^{2}$, which for $\mathrm{CAB}$ at $\Delta v=1 / 6$ gives a $17.4 \mathrm{~dB}$ reduction.

To summarize the analysis, we anticipate a substantial reduction $(5.7 \mathrm{~dB})$ in shot noise and large reduction $(17.4 \mathrm{~dB})$ in intensity noise with $\mathrm{CAB}$ operation at the $\Delta v=1 / 6$ bias point, where signal powers are equal.

\section{EXPERIMENT}

As shown in Figure 2, a tunable laser was coupled to an erbium-doped fiber amplifier (EDFA) (17 dBm output power) and a tunable filter (TF) to minimize 
spontaneous-spontaneous beat noise. The two matched intensity modulators (JGKB Photonics, Inc.) were biased in a complementary configuration with a maximum of 12 $\mathrm{dBm}$ optical power available at each input after passing through a $3 \mathrm{~dB}$ coupler and a polarization controller (PC).

The balanced detector (Discovery Semiconductors, Inc.) consisted of two $17 \mathrm{GHz}$ InGaAs detectors with a specified common-mode suppression of $30 \mathrm{~dB}$. Performance comparisons were made between two link designs - QB and CAB. For QB, the fiber was simply disconnected from $\mathrm{D} 2$ and the bias point adjusted to quadrature. To determine $R I N^{*}$, noise was measured for the highest optical power level with no modulation. Results were consistent with an $R I N^{*}$ of $-154.2 \mathrm{~dB} / \mathrm{Hz}$. Modulation was applied to the modulators from two sweep generators (1.98 and $2.02 \mathrm{GHz}$ ).

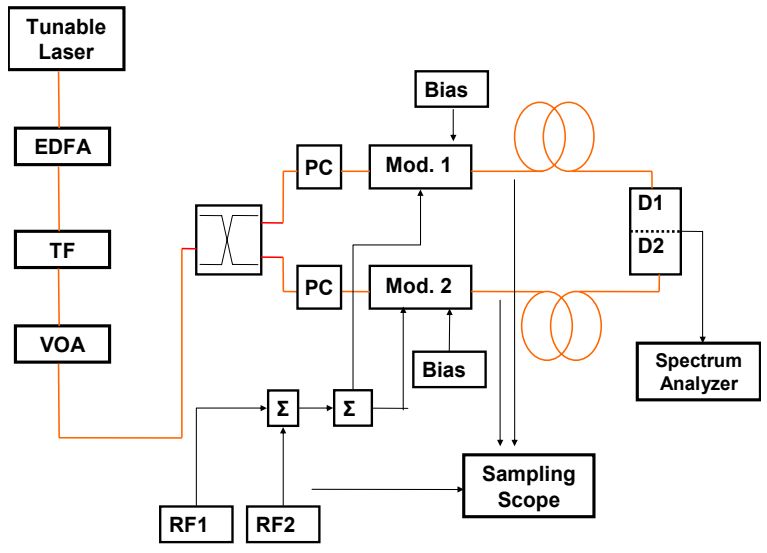

Fig. 2. Experimental setup for tests using single or complementary modulators.

The CAB link requires matching of several parameters for reasonable reconstruction of the signal. These include modulation depth, phase of the RF and optical signals in the two paths, bias points on the modulators, and the detected optical powers. A sampling oscilloscope was used to match phase and attenuators were placed in the RF and optical paths to balance the RF and optical powers. Bias points were controlled manually. In order to bias the modulators for $\mathrm{CAB}$ operation, transmission at QB was determined by observing the bias voltage at which the second-harmonic distortion was minimized. At the desired bias point, $V_{\pi} / 6$ away from zero transmission, the DC transmission is 0.134 times the transmission at $V_{\pi} / 2$.

SFDR measurements were automated through the IEEE 488 interface on the sweep generators and the RF spectrum analyzer. Third-order distortion analysis was done for the highest available optical power for the two link configurations, to determine the SFDR.

\section{RESULTS AND DISCUSSION}

Predicted and measured signal and noise are presented in Figure 3, for $\mathrm{QB}$ and $\mathrm{CAB}$ (with $\Delta v=1 / 6$ ). Agreement between theory and experiment is excellent, showing equal RF power at these two bias points, but with the predicted reduction in noise anticipated given the reduction in total received DC power.

Due to limited available optical power and the lowbias operation, we were unable to extend the total $\mathrm{CAB}$ DC power levels to those realized with $\mathrm{QB}$. Therefore overall noise levels, particularly for $\mathrm{CAB}$, were close to receiver and analyzer noise. Results show noise levels including shot, RIN*, receiver, and analyzer noise, and those corrected by subtracting the measured RIN*, receiver, and analyzer noise powers. For CAB, RIN ceases to be a concern.

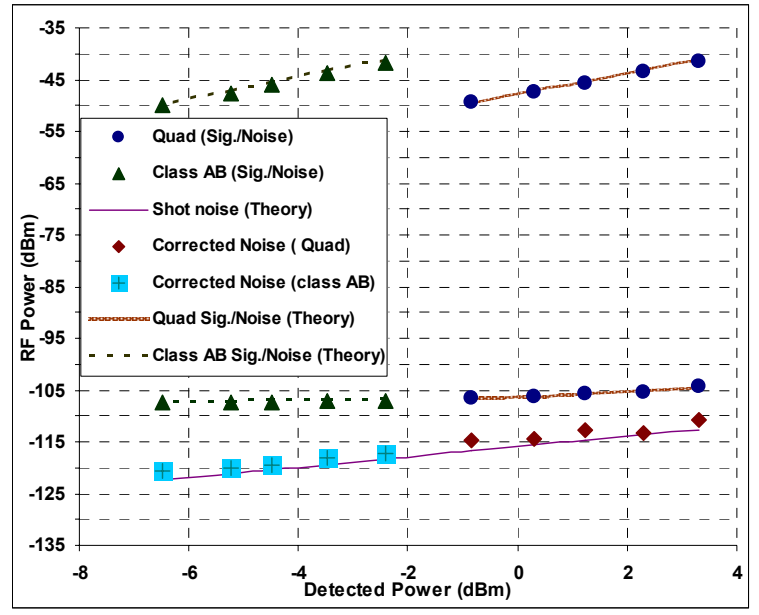

Fig. 3. Results from experiment (points) and theory (lines) for Q-biased and Class-AB links versus total received optical power. Noise is referenced to a $1 \mathrm{MHz}$ bandwidth.

Figure 4 shows the measured signal, noise, and 2-tone $3^{\text {rd }}$-order intermodulation distortion for the two links. Again, the signal power is identical for $\mathrm{QB}$ and $\mathrm{CAB}$. Also, there is no significant difference in $3^{\text {rd }}$-order distortion, confirming that the $\mathrm{CAB}$ configuration has successfully re-created the odd-order components of the identical sinusoidal transfer function to QB. Secondorder distortion is a stronger function of exact balance and bias, and cannot be studied meaningfully without active bias control (future work). CAB provides an improvement in SFDR, from 102.3 dB for QB to 104.0 $\mathrm{dB}$ for $\mathrm{CAB}$ (including receiver and analyzer noise) and from $105.1 \mathrm{~dB}$ for $\mathrm{QB}$ to $110 \mathrm{~dB}$ for $\mathrm{CAB}$, correcting for receiver and analyzer noise.

SFDR could be improved for both links using a $3^{\text {rd }}$ order linearization technique. In addition, SFDR could be improved by increasing the optical power. For QB, more power would result eventually in additional intensity noise, and challenges related to fiber nonlinearity and detector saturation. For $\mathrm{CAB}$ operation with the same total optical power as QB, the signal power is $12 \mathrm{~dB}$ larger (Figure 3 and Equation (6)). Also, 
since power for $\mathrm{CAB}$ is divided between two detectors, $\mathrm{CAB}$ could operate at twice the total power as QB for an additional $6 \mathrm{~dB}$ in signal power.

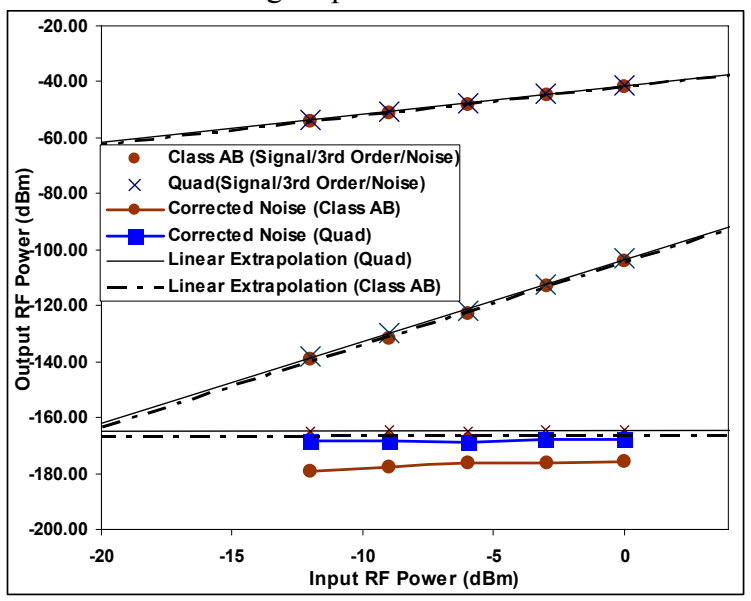

Fig. 4. Experimental results for spur-free dynamic range measurement using Q-biased and Class-AB links. Noise is referenced to a $1 \mathrm{HZ}$ bandwidth. Linear extrapolations are least-squares fit, based on measured signal, $3^{\text {rd }}$-order distortion, and total noise power.

We would have preferred to compare $\mathrm{CAB}$ with a balanced QB (BQB) link in which noise from RIN* was suppressed in the balanced detector. However, our modulators have only one output fiber. For a BQB link, intensity noise would be suppressed by some factor dependent on the modulation index [13]. Signal power would have been increased by $6 \mathrm{~dB}$ relative to the single QB link, with a $3 \mathrm{~dB}$ increase in shot noise (same as doubling optical power in a single QB link). Therefore, if limited by transmit power, $\mathrm{CAB}$ would have resulted in a $2.7 \mathrm{~dB}$ reduction in shot noise relative to $\mathrm{BQB}$. More importantly, if limited by detector saturation or fiber nonlinearity, $\mathrm{CAB}$ could allow operation at $12 \mathrm{~dB}$ higher modulated signal powers than $\mathrm{BQB}$.

\section{SUMMARY}

We have demonstrated the efficacy of Class-AB techniques in minimizing carrier-related noise in microwave-photonic links. By direct comparison with a conventional quadrature-biased link, results predict and demonstrate substantial $(5.7 \mathrm{~dB})$ reduction in shot noise and the elimination (predict $17 \mathrm{~dB}$ reduction) of intensity noise, while retaining the same $3^{\text {rd }}$-order distortion. We feel that this approach opens an attractive new pathway to higher-SFDR optical links.

\section{ACKNOWLEDGEMENT}

The authors wish to acknowledge the support of National Sciences and Engineering Research Council,
Canadian Institute for Photonic Innovation, and Discovery Semiconductors.

\section{REFERENCES}

[1] C. H. Cox III, G. E. Betts, and L. M. Johnson, “An analytic and experimental comparison of direct and external modulation in analog fiber-optic links," IEEE Trans. Microwave Theory and Techniques, vol. 38, pp. 501-509, May 1990.

[2] L. T. Nichols, K. J. Williams, and R. D. Esman, "Optimizing the ultrawide-band photonic link," IEEE Trans. Microwave Theory and Techniques, vol. 45, pp. 1384-1389, August 1997.

[3] C. H. Cox, III, "Analog Optical Links: Theory and Practice", Cambridge, 2004.

[4] J. M. P. Delavaux, A Yeniay, B Neyret, C. Hullin, G. R. Wilson, "Multiple-output Er-3+ amplifier for analog and QAM distribution systems," Optical Fiber Communications Conference, 2001, Vol. 3, Page(s) WDD30-1-3.

[5] X. P. Mao, G. E. Bodeep, R. W. Tkach, A. R. Chraplyvy, T. E. Darcie, R. M. Derosier, "Brillouin scattering in externally modulated lightwave AMVSB transmission systems," IEEE Photonics Technol. Lett., Vol. 4, Issue 3, March 1992, Page(s) 287-289.

[6] M. R. Phillips, T. E. Darcie, D. Marcuse, G. E. Bodeep, N. J. Frigo, "Nonlinear distortion generated by dispersive transmission of chirped intensity-modulated signals," IEEE Photonics Technol. Lett., Volume 3, Issue 5, May 1991 Page(s): $481-483$.

[7] R. D. Esman, K. J. Williams, "Wideband efficiency improvement of fiber optic systems by carrier subtraction," IEEE Photonics Technol. Lett., Vol. 7, No. 2, Feb. 1995 Page(s): $218-220$

[8] A. C. Lindsay, "An analysis of coherent carrier suppression for photonic microwave links," IEEE Trans. Microwave Theory and Tech., Vol. 47, Issue 7, July 1999 Page(s): 1194 - 1200

[9] T. E. Darcie, P. P. Ianonne, "Method and apparatus for laser performance enhancement," US patent \#6,181,453, January 30, 2001.

[10] S. Mathai, F. Cappelluti, T. Jung, D. Novak, R. B. Waterhouse, D. Sivco, A. Y. Cho, G. Ghione, M. C. $\mathrm{Wu}$, "Experimental demonstration of a balanced electroabsorption modulated microwave photonic link, "IEEE Trans. Microwave Theory and Tech., vol. 49, pp. 1956-1961, Oct. 2001.

[11] W. K. Burns, G. K. Gopalakrishnan, R.P Moeller, "Multi-octave operation of low-biased modulators by balanced detection," IEEE Photonics Technol. Lett., Volume 8, Issue 1, Jan. 1996, Page(s): 130 132

[12] C. Trask, "High efficiency broadband linear pushpull power amplifiers using linearity augmentation," IEEE International Symposium on Circuits and Systems, ISCAS 2002, Volume 2, 26-29 May, 2002, Page(s): II-432 - II-435 .

[13] T. E. Darcie, A Moye, "Modulation-dependent limits to intensity-noise suppression in modulated microwave-photonic links," submitted to IEEE Photonics Technol. Lett., April 2005. 